Quim. Nova, Vol. 26, No. 6, 934-937, 2003

\title{
ELETRODEPOSIÇÃO DE IRÍDIO EM TUBO DE GRAFITE COMO MODIFICADOR QUÍMICO PERMANENTE EM ESPECTROMETRIA DE ABSORÇÃO ATÔMICA
}

\author{
Juliana Naozuka e Pedro V. Oliveira* \\ Instituto de Química, Universidade de São Paulo, CP 26077, 05513-970 São Paulo - SP \\ Jairo J. Pedrotti \\ Departamento de Química, Faculdade de Ciências Biológicas, Exatas e Experimentais, Universidade Presbiteriana Mackenzie, \\ 01239-900 São Paulo - SP
}

Recebido em 21/11/02; aceito em 19/3/03

\begin{abstract}
ELECTRODEPOSITION OF IRIDIUM IN GRAPHITE TUBE AS PERMANENT CHEMICAL MODIFIER IN ATOMIC ABSORPTION SPECTROMETRY. A tubular electrochemical flow-cell for iridium deposition on the inner surface of pyrolytic graphite tube for permanent chemical modification is proposed. A transversal heated graphite tube was used as working electrode, a cylindrical piece of graphite inserted into the graphite tube as auxiliary electrode, and a micro $\mathrm{Ag} / \mathrm{AgCl}_{\text {(sat) }}$ as reference electrode. Iridium solution in $1.0 \mathrm{~mol} \mathrm{~L}^{-1} \mathrm{HCl}$, flowing at $0.55 \mathrm{~mL} \mathrm{~min}^{-1}$ for $60 \mathrm{~min}$ was used to perform the electrochemical modification. The applied potential to the flow-cell was $-0.700 \mathrm{~V} v \mathrm{Ag} / \mathrm{AgCl}$. Scanning electron microscopy images were taken for thermal and electrochemical modified graphite surface in order to evaluate the iridium distribution. Selenium hydride trapping was used to verify the performance of the proposed permanent chemical modifier.
\end{abstract}

Keywords: iridium electrodeposition; permanent chemical modifier; atomic absorption spectrometry.

\section{INTRODUÇÃO}

O uso de modificador químico é uma prática freqüentemente utilizada nas determinações por espectrometria de absorção atômica com atomização eletrotérmica (ETAAS) ${ }^{1,2}$. Na maioria das aplicações, o papel do modificador químico é estabilizar termicamente o analito, possibilitando o emprego de temperaturas mais elevadas durante a etapa de pirólise para reduzir concomitantes e/ou melhorar as vias de atomização do analito ${ }^{3}$.

Em geral, uma alíquota da solução do modificador químico é introduzida no tubo de grafite juntamente com a solução analítica. Por outro lado, uma pequena área da superfície interna do tubo de grafite pode ser modificada com um elemento metálico que atua como modificador químico permanente, o qual, dependendo da temperatura de limpeza adotada no programa de aquecimento, pode suportar vários ciclos de aquecimento.

Além do uso para soluções aquosas e suspensões, o modificador químico permanente ampliou as aplicações da ETAAS para a determinação de elementos formadores de espécies voláteis como o mercúrio na geração de vapor a frio (CV-ETAAS) ${ }^{4-7}$ e os hidretos na geração de hidretos (HG-ETAAS) ${ }^{8-10}$. Os vapores gerados quimicamente são conduzidos, por um fluxo de um gás inerte, para a parede modificada do tubo de grafite. Além da separação da matriz, a concentração do analito na superfície do tubo de grafite é uma característica importante do sistema.

Entre os procedimentos de modificação química da superfície do tubo de grafite, o processo térmico predomina sobre o eletroquímico $^{4,7}$. Em parte, isso pode ser explicado devido a aparente dificuldade atribuída ao processo de eletrodeposição, ou a falta de instrumentação disponível para executá-lo. No entanto, com o processo eletroquímico é possível recobrir grande parte da superfície interna

*e-mail: pvolivei@iq.usp.br do tubo de grafite com o elemento metálico de interesse, melhorando o limite de detecção e aumentando a vida útil do filme metálico ${ }^{4}$. Além disso, o modificador químico permanente obtido por processo eletroquímico vem apresentando melhor eficiência no trapeamento de espécies voláteis como $\mathrm{Hg}$ e hidretos, quando comparado com aqueles preparados usando o processo térmico. Uma hipótese para explicar este melhor desempenho é o aumento da área superficial.

Os modelos de células eletroquímicas para a modificação dos tubos de grafite utilizam preferencialmente sistemas em batelada ${ }^{11-14}$. Em geral, este tipo de arranjo utiliza corrente controlada e o tubo de grafite necessita ser revestido externamente com material protetor para evitar o recobrimento metálico. Esta estratégia foi utilizada para eletrodeposição de $\mathrm{Pd}$ e $\mathrm{Rh}^{11}, \mathrm{Au}, \mathrm{Ir}, \mathrm{Pd}$ e $\mathrm{Rh}^{12}, \mathrm{Cu}, \mathrm{Ni}$ e $\mathrm{Pd}^{13}$ e $\operatorname{Ir}^{14}$ com uma célula eletroquímica formada por um fio de Pt (anodo) e um tubo de grafite (catodo), operando a corrente controlada.

Nas aplicações desses modificadores, o filme de Pd e Rh eletrodepositado mostrou-se bastante eficiente na estabilização térmica de As e Se, bem como na redução da formação de óxidos e carbetos de $\mathrm{Si}^{11}$. Entre os modificadores químicos permanentes $\mathrm{Au}, \mathrm{Au} / \mathrm{Rh}, \mathrm{Ir}$, $\mathrm{Pd}$ e Rh utilizados para a determinação de $\mathrm{Hg}$, os melhores resultados foram com $\mathrm{Pd}$ e a mistura $\mathrm{Au} / \mathrm{Rh}$, tanto para aqueles obtidos por processos térmicos como eletroquímicos ${ }^{12}$. Na determinação de diferentes espécies de selênio por ETAAS com tubo modificado permanentemente com $\mathrm{Cu}, \mathrm{Ni}$, ou Pd, o filme de Pd eletrodepositado foi o mais eficiente ${ }^{13}$. Em todos esses casos, os modificadores químicos obtidos por processos eletroquímicos apresentaram tempo de vida similar ou superior ao daqueles obtidos por processos térmicos.

Recentemente, modelos de células eletroquímicas em fluxo foram propostos para a modificação da superfície de tubos de grafite com Pd usando potencial ${ }^{4,5}$ ou corrente controlada ${ }^{15}$. Em comparação com as células em batelada, a renovação constante da solução na superfície grafítica torna o transporte do material eletroativo mais eficiente nas células em fluxo. Entretanto, o processo a potencial controlado permite maior seletividade e possibilita a formação de um depósito mais uniforme e aderente ${ }^{4,5}$. 
A necessidade de condicionamento térmico do tubo de grafite após a eletrodeposição é imperativa, tanto para aumentar o tempo de vida útil do filme de Pd, quanto para melhorar a precisão dos resultados analíticos. Os tubos de grafite modificados com Pd foram utilizados na determinação de $\mathrm{Hg}$ em amostras ambientais por geração de vapor a frio ${ }^{4}$ e em carvão, por um processo que envolve a transferência do analito da plataforma para a superfície modificada ${ }^{15}$.

A célula eletroquímica anteriormente proposta para eletrodeposição de $\mathrm{Pd}^{4,5}$ não apresentou um bom desempenho para a eletrodeposição de Ir. O posicionamento e o material do eletrodo auxiliar foram aspectos determinantes. Dentro desse contexto, o trabalho proposto apresenta uma célula eletroquímica robusta e de fácil construção, bem como um procedimento para a modificação química permanente da parede interna de tubos de grafite com Ir para ETAAS. O trapeamento de hidreto de selênio foi utilizado como modelo para verificar as características do modificador proposto.

\section{PARTE EXPERIMENTAL}

\section{Instrumentação}

Um espectrômetro de absorção atômica com atomização eletrotérmica ZEEnit 60 (AnalytikJena AG, Alemanha), equipado com lâmpada de catodo oco, tubo de grafite com aquecimento transversal e corretor de radiação de fundo baseado no efeito Zeeman foi utilizado durante todo o trabalho. As medidas de absorbância de selênio foram feitas em 196,0 nm. A corrente adotada para a lâmpada de catodo oco de selênio foi de $4 \mathrm{~mA}$.

Os estudos voltamétricos para encontrar as melhores condições de eletrodeposição de Ir foram efetuados com um potenciostato PAR 273 A da marca EG\&G (Princeton Applied Research, USA) interfaceado a um microcomputador AT DX $40 \mathrm{MHz}$.

A Figura 1 ilustra a célula eletroquímica utilizada para a modificação da superfície interna do tubo de grafite. A configuração dos eletrodos foi alterada em relação a um modelo anteriormente proposto por Moreno et al. ${ }^{4,5}$. Um mini eletrodo de referência de Ag/ $\mathrm{AgCl}$ (saturado com $\mathrm{KCl}$ ) foi posicionado na entrada da célula eletroquímica com o auxílio de um suporte de acrílico. $\mathrm{O}$ tubo de grafite, com $1,7 \mathrm{~cm}$ de comprimento e $0,5 \mathrm{~cm}$ de diâmetro interno, foi o eletrodo de trabalho. Na extremidade oposta do tubo de grafite, posicionou-se o eletrodo auxiliar, constituído de um tarugo cilíndrico de grafite pirolítico $(1=3,0 \mathrm{~cm}$ e $\mathrm{d}=0,6 \mathrm{~cm})$ sobre o qual foi confeccionado um canal central $(1=1,8 \mathrm{~cm} \mathrm{e} \mathrm{d}=1,0 \mathrm{~mm})$ para permitir a saída da solução. Uma parte desse tarugo $(1=1,5 \mathrm{~cm}$ e d $=$ $0,3 \mathrm{~cm}$ ) foi torneada de maneira a permitir encaixe na extremidade do tubo de grafite e o posicionamento no seu interior (Figura 1). Os dois eletrodos (trabalho e auxiliar) foram fixados entre si com auxílio de um anel de silicone. Para evitar vazamento de solução, o orifício sobre o tubo de grafite $(\mathrm{d}=0,2 \mathrm{~cm})$, originalmente projetado

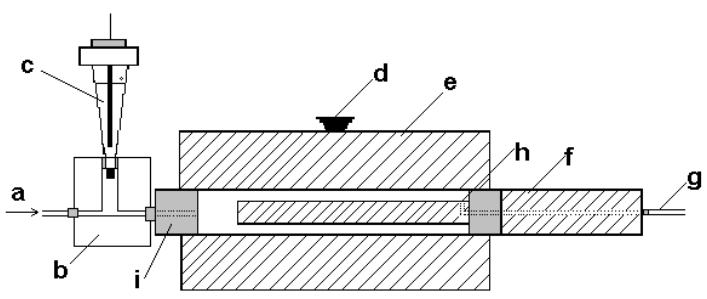

Figura 1. Representação esquemática da célula eletroquímica de fluxo: (a) entrada de solução; (b) suporte de acrílico; (c) eletrodo de referência; (d) rolha de PVC; (e) eletrodo de trabalho (tubo de grafite); (f) eletrodo auxiliar; (g) tubo de polietileno; (h) saída de solução; (i) tubo de silicone para introdução de soluções, foi vedado com cilindro maciço de policloreto de vinila (PVC), recoberto com fita de politetrafluoretileno (PTFE).

O controle do potencial na célula eletroquímica foi assegurado com o uso de um potenciostato de baixo custo com compliância de potencial de $\pm 11 \mathrm{~V}$, desenvolvido no laboratório dos autores. Os valores dos potenciais aplicados ao eletrodo de trabalho e o valor de sinal de corrente catódica foram monitorados com um voltímetro PROTEK modelo 233 de $3^{1 / 2}$ dígitos (resolução de $\pm 1 \mathrm{mV}$ ).

O fluído transportador foi impulsionado através da célula por uma bomba peristáltica modelo MS-REGLO (ISMATEC, Suíça). O tempo de deposição para formação do filme de Ir foi controlado manualmente (aproximadamente $60 \mathrm{~min}$ ). Todas as eletrodeposições foram efetuadas sob temperatura ambiente $\left(25 \pm 2{ }^{\circ} \mathrm{C}\right)$.

Um microscópio eletrônico de varredura (MEV) LCT Stereoscan 440 (LEO, Inglaterra) foi utilizado para a inspeção da superfície do tubo de grafite.

\section{Reagentes e soluções}

Todas as soluções foram preparadas empregando-se água destilada e desionizada em sistemas de purificação Milli-Q ${ }^{\circledR}(18 \mathrm{M} \Omega \mathrm{cm})$ (Millipore, USA). Os ácidos nítrico e clorídrico utilizados na preparação das soluções de referência foram purificados em subdestiladores (Marconi S/A, Brasil). Toda a vidraria e recipientes para armazenamento das soluções foram lavados com detergente neutro, imersos em solução de ácido nítrico $10 \% \mathrm{v} \mathrm{v}^{-1}$ e enxaguados com água desionizada antes do uso.

Solução contendo $1000 \mathrm{mg} \mathrm{L}^{-1}$ de $\mathrm{Ir}^{4+}\left(\mathrm{Na}_{2} \mathrm{IrCl}_{6}\right)$ em 1,00 mol L-1 de $\mathrm{HCl}$ foi utilizada para a modificação eletroquímica da superfície interna do tubo de grafite. Para a modificação térmica foi utilizada solução de $1000 \mathrm{mg} \mathrm{L}^{-1}$ de $\mathrm{Ir}^{4+}$ em meio de $0,1 \% \mathrm{v} \mathrm{v}^{-1} \mathrm{HNO}_{3}$.

As soluções analíticas de referência de selênio foram preparadas a partir de diluições sucessivas de $1000 \mathrm{mg} \mathrm{L}^{-1}$ de Se $\mathrm{Se}^{4+}$ (Merck, Alemanha).

Solução contendo $0,15 \mathrm{~mol} \mathrm{~L}^{-1} \mathrm{NaBH}_{4}$ (Nuclear p.a.) em $0,1 \mathrm{~mol} \mathrm{~L}^{-1}$ de $\mathrm{NaOH}$ (Merck p.a.) foi utilizada como redutora para a geração de $\mathrm{SeH}_{2}$.

\section{Procedimento}

A eletrodeposição de Ir sobre a superfície do tubo de grafite foi feita com a célula eletroquímica em fluxo ilustrada na Figura 1, sob vazão de $0,55 \mathrm{~mL} \mathrm{~min}{ }^{-1}$, com recirculação da solução de $\mathrm{Ir}^{4+}$ durante $1 \mathrm{~h}$, empregando potenciais de $-0,700 \mathrm{~V}$ vs $\mathrm{Ag} / \mathrm{AgCl}$. Após a eletrodeposição, o tubo de grafite foi posicionado no forno de grafite e submetido a um programa de aquecimento para condicionamento térmico (temperatura, rampa, patamar): $110^{\circ} \mathrm{C}, 30{ }^{\circ} \mathrm{C} \mathrm{s}^{-1}, 15 \mathrm{~s}$; $130{ }^{\circ} \mathrm{C}, 30{ }^{\circ} \mathrm{C} \mathrm{s}^{-1}, 10 \mathrm{~s} ; 800{ }^{\circ} \mathrm{C}, 4{ }^{\circ} \mathrm{C} \mathrm{s}^{-1}, 10 \mathrm{~s} ; 2500{ }^{\circ} \mathrm{C}, 50{ }^{\circ} \mathrm{C} \mathrm{s}^{-1}, 5 \mathrm{~s}$.

O programa de aquecimento para a modificação térmica com Ir foi (temperatura, rampa, patamar): $100{ }^{\circ} \mathrm{C}, 10{ }^{\circ} \mathrm{C} \mathrm{s}^{-1}, 30 \mathrm{~s} ; 150{ }^{\circ} \mathrm{C}$, $50{ }^{\circ} \mathrm{C} \mathrm{s}^{-1}, 10 \mathrm{~s} ; 1200{ }^{\circ} \mathrm{C}, 50{ }^{\circ} \mathrm{C} \mathrm{s}^{-1}, 5 \mathrm{~s} ; 100{ }^{\circ} \mathrm{C}, 20^{\circ} \mathrm{C} \mathrm{s}^{-1}, 2 \mathrm{~s} ; 2500{ }^{\circ} \mathrm{C}$, $1200{ }^{\circ} \mathrm{C} \mathrm{s}^{-1}, 10 \mathrm{~s}^{16}$. Alíquotas de $40 \mu \mathrm{L}$ de solução contendo

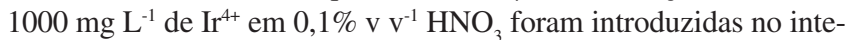
rior do tubo de grafite e o programa de aquecimento foi executado. Essa operação foi realizada em triplicata, de maneira que a massa final de Ir depositado na superfície do tubo de grafite foi de $120 \mu \mathrm{g}$.

O sistema de geração de hidretos foi otimizado com uma solução de $0,50 \mu \mathrm{g} \mathrm{L}^{-1}$ de $\mathrm{Se}^{4+}$ em 1,2 mol L ${ }^{-1}$ de $\mathrm{HCl}$. Um volume de $1,00 \mathrm{~mL}$ de solução analítica de $\mathrm{Se}^{4+}$ reagiu com $1,00 \mathrm{~mL}$ da solução de $\mathrm{NaBH}_{4}$, formando o hidreto volátil que foi transportado do reator/ separador gás-líquido por um gás inerte (argônio, $300 \mathrm{~mL} \mathrm{~min}^{-1}$ ) até a superfície do tubo de grafite modificada com Ir. O sistema de gera- 
ção de hidretos utilizado foi confeccionado no laboratório dos autores ${ }^{4,5}$. O tempo de concentração adotado foi de $60 \mathrm{~s}$.

O programa de aquecimento utilizado para atomização de selênio foi (temperatura, rampa, patamar): secagem I - $120{ }^{\circ} \mathrm{C}, 30{ }^{\circ} \mathrm{C} \mathrm{s}^{-1}$, $10 \mathrm{~s}$; secagem II $-130{ }^{\circ} \mathrm{C}, 10{ }^{\circ} \mathrm{C} \mathrm{s} \mathrm{s}^{-1}, 5 \mathrm{~s}$; atomização $-2200{ }^{\circ} \mathrm{C}$, $2200{ }^{\circ} \mathrm{C} \mathrm{s}^{-1}, 5 \mathrm{~s}$; limpeza $-2300{ }^{\circ} \mathrm{C}, 2200{ }^{\circ} \mathrm{C} \mathrm{s}^{-1}, 2 \mathrm{~s}$.

\section{RESULTADOS E DISCUSSÃO}

A otimização do sistema para eletrodeposição de Ir foi efetuada a partir de estudos voltamétricos com solução de $1000 \mathrm{mg} \mathrm{L}^{-1}$ de $\mathrm{Ir}^{4+}$ em 1,00 mol L ${ }^{-1}$ de $\mathrm{HCl}$, utilizado como eletrólito suporte. Esse eletrólito assegurou uma concentração hidrogeniônica livre na solução capaz de evitar a hidrólise do $\mathrm{Ir}^{4+}$, bem como de estabelecer um limite adequado do intervalo de potencial do eletrodo de grafite pirolítico $(+1,00 \mathrm{~V} \mathrm{a}-0,80 \mathrm{~V})$. $\mathrm{O}$ valor do potencial de trabalho selecionado $(-0,700 \mathrm{~V}$ vs $\mathrm{Ag} / \mathrm{AgCl})$ corresponde a região de potencial em que se assegura a redução do $\operatorname{Ir}^{4+}$ a $\operatorname{Ir}^{0}$, sem que ocorra a descarga significativa dos íons $\mathrm{H}^{+}$.

Na Figura 2 estão apresentadas as imagens, obtidas por microscopia eletrônica de varredura, das superfícies grafíticas modificadas termicamente (Figura 2a) e eletroquimicamente (Figura $2 \mathrm{~b}$ e 2c) com Ir. Em todos os casos, os aglomerados, representados pelas regiões claras das imagens, foram qualitativamente avaliados por espectroscopia eletrônica dispersiva (EDS), indicando a presença de $\mathrm{Ir}^{0}$. Sendo assim, as regiões escuras representam o grafite pirolítico, enquanto que as claras o $\operatorname{Ir}^{0}$.

$\mathrm{Na}$ imagem da superfície modificada por processo térmico observam-se pequenos aglomerados, os quais estão distribuídos uniformemente na região em que foi depositada a alíquota da solução de $\operatorname{Ir}^{4+}$ (Figura 2a).

A imagem da superfície após a eletrodeposição revelou a presença de grandes aglomerados de $\operatorname{Ir}^{0}$ distribuídos aleatoriamente sobre a superfície do tubo de grafite (Figura 2b). Imagens de outras regiões da superfície do tubo de grafite revelaram aspecto semelhante variando entre grandes, médios e pequenos aglomerados. A maior quantidade de grandes aglomerados é um indicativo da deposição preferencial de $\mathrm{Ir}^{0}$ sobre $\mathrm{Ir}^{0}$.

A microscopia eletrônica da parede interna do tubo de grafite após sofrer eletrodeposição e pré-condicionamento térmico revelou uma distribuição uniforme de $\operatorname{Ir}^{0}$ (Figura 2c), aumentando consideravelmente a superfície de contato, quando comparado com a eletrodeposição (Figura 2b).

Durante o pré-condicionamento térmico a temperatura máxima atingida foi de $2500{ }^{\circ} \mathrm{C}$, o suficiente para promover a fusão $(\mathrm{PF}=$ $\left.2410^{\circ} \mathrm{C}\right)^{16}$ mas não a vaporização do $\operatorname{Ir}^{0}\left(\mathrm{PE}=4527{ }^{\circ} \mathrm{C}\right)^{16}$, espalhando-o para as regiões do tubo de grafite onde não ocorreu eletrodeposição. Além disso, a partir das medidas por EDS foi possível observar que o pré-condicionamento eliminou contaminações de espécies contendo cloro, oxigênio e sódio, as quais foram incorporadas aos aglomerados de $\operatorname{Ir}^{0}$ durante a eletrodeposição.

Para testar o desempenho do tubo modificado com $\mathrm{Ir}^{0}$, usando processo eletroquímico + pré-condicionamento, e comparar com um modificado termicamente utilizou-se um sistema para geração de hidretos de selênio. A avaliação dos resultados das massas características e limites de detecção para Se mostra um melhor desempenho para o modificador químico obtido por processo eletroquímico $\left(m_{o}\right.$ $\left.=43 \mathrm{pg} ; \mathrm{LD}=0,11 \mu \mathrm{g} \mathrm{L} \mathrm{L}^{-1}, \mathrm{n}=10\right)$ do que para aquele obtido por processo térmico $\left(m_{o}=60 \mathrm{pg} ; \mathrm{LD}=0,18 \mu \mathrm{g} \mathrm{L}{ }^{-1}, \mathrm{n}=10\right)$. Esse efeito pode estar associado ao aumento da área superficial modificada no processo eletroquímico $\left(2,04 \mathrm{~cm}^{2}\right)$ em comparação com o térmico $\left(0,57 \mathrm{~cm}^{2}\right)$, o que melhora a eficiência no trapeamento do hidreto de selênio.
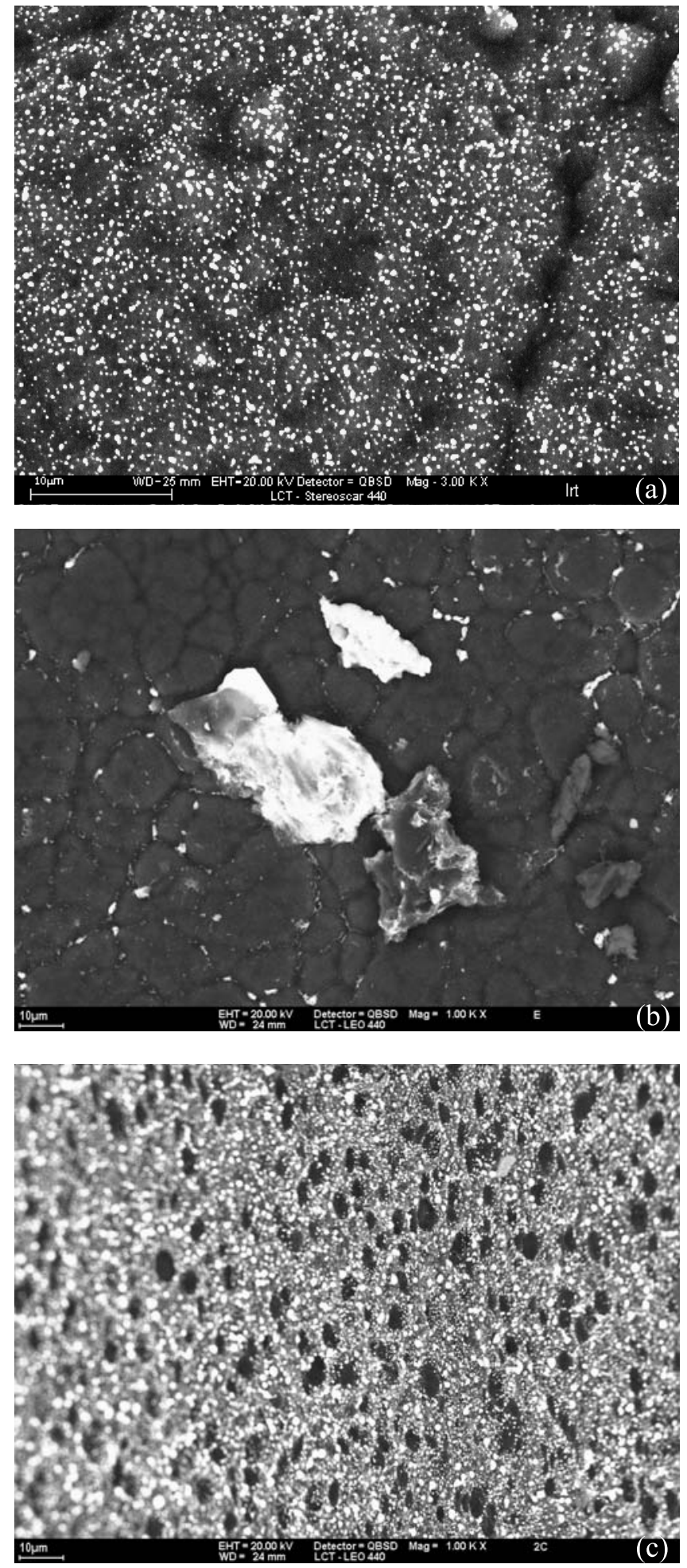

Figura 2. Microscopia eletrônica de varredura da parede interna de tubos de grafite com superfície modificada com Ir $^{0}$, usando processo térmico (ampliação 3000x): (a) e eletroquímico antes (b) e depois (c) do condicionamento térmico (ampliação 1000x)

\section{CONCLUSÕES}

A célula eletroquímica proposta é simples, robusta e muito prática para a modificação de uma grande superfície interna do tubo de grafite. A geometria e a grande área do eletrodo auxiliar asseguram 
melhor distribuição das linhas de potencial sobre o eletrodo de trabalho, o que garante uma melhor deposição do metal sobre a superfície do tubo de grafite. O sistema permite a modificação de tubos de grafite com diferentes geometrias.

\section{AGRADECIMENTOS}

Os autores agradecem à Fundação de Amparo à Pesquisa do Estado de São Paulo (FAPESP) pelo apoio financeiro (Processo 2001/ 07048-1). J. Naozuka agradece ao Conselho Nacional de Desenvolvimento Científico e Tecnológico pelas bolsas de iniciação científica (CNPq/PIBIC) e mestrado (CNPq).

\section{REFERENCIAS}

1. Welz, B.; Sperling, M.; Atomic Absorption Spectrometry, $3^{\text {rd }}$ ed., WileyVCH: Weinheim, 1999.

2. Jackson, K. W. ed.; Electrothermal Atomization for Analytical Atomic Spectrometry, John Wiley \& Sons Ltd: Chichester, 1999.
3. Tsalev, D. L.; Slaveykova, V. I.; Lampugnani, L.; D’Ulivo, A.; Georgieva, R.; Spectrochim. Acta 2000, 55B, 473.

4. Moreno, R. G. M.; Oliveira, E.; Pedrotti, J. J.; Oliveira, P. V.; Spectrochim. Acta 2002, 57B, 769

5. Moreno, R. G. M.; Oliveira, E.; Pedrotti, J. J.; Oliveira, P. V.; Quim. Nova 2001, 24, 404

6. Moreda-Piñeiro, J.; López-Mahía, P.; Muniategui-Lorenzo, S.; FernándezFernández, E.; Prada-Rodríguez, D.; Anal. Chim. Acta 2002, 460, 111.

7. Bulska, E.; Thybusch, B.; Ortner, H. M.; Spectrochim. Acta 2001, 56B, 363.

8. Ringmann, S.; Boch, K.; Marquardt, W.; Schuster, M.; Schlemmer, G.; Kainrath, P.; Anal. Chim. Acta 2002, 452, 207.

9. Moreda-Piñeiro, J.; López-Mahía, P.; Muniategui-Lorenzo, S.; FernándezFernández, E.; Prada-Rodríguez, D.; Anal. Chim. Acta 2002, 461, 261.

10. Tsalev, D. L.; Spectrochim. Acta 2000, 55B, 917.

11. Bulska, E.; Jedral, W.; J. Anal. At. Spectrom. 1995, 10, 49.

12. Bulska, E.; Kandler, W.; Hulannicki, A.; Spectrochim. Acta 1996, 51, 1263.

13. Buska, E.; Pyrznska, K.; Spectrochim. Acta 1997, 52B, 1283.

14. Buska, E; Piascik, M.; Pyrzynska, K.; Anal. Lett. 2000, 33, 1399.

15. Maia, M. M.; Welz, B.; Edgard, G.; Curtius, J. A.; Spectrochim. Acta 2002, 57,473

16. Vassileva, E.; Baeten, H.; Hoenig, M.; Fres. J. Anal. Chem. 2001, 369, 491. 